\title{
Evaluación del método Scenario Centered Curriculum en función del perfil tecnológico del estudiante
}

\section{Scenario Centered Curriculum Assessment Based on the Technological Profile of the Student}

\author{
David Fonseca Escudero ${ }^{1}$, August Climent ${ }^{1}$, Xavier Canaleta ${ }^{1}$, Lluís Vicent ${ }^{2}$
}

\begin{abstract}
'Department of Engineering and Group of Research in Technology Enhanced Learning (GRETEL), La Salle, Universitat Ramon Llull, Barcelona. Spain.\{fonsi, augc, xavic\}@salle.url.edu

Group of Research in Technology Enhanced Learning (GRETEL), La Salle, Universitat Ramon LIull, Barcelona, Spain, and UOLS, Andorra la Vella,Andorra. vicent@salle.url.edu
\end{abstract}

\section{Resumen}

En el presente artículo se presentan los resultados obtenidos de evaluar la usabilidad del método Scenario Centered Curriculum (SCC) y el grado de satisfacción de los estudiantes con el mismo, en función del perfil tecnológico de cada grupo de estudiantes. El método se ha introducido en un curso de Marketing Digital impartido en tres escuelas de Formación Profesional de España, Italia y Francia. Dichas escuelas forman parte, junto a otros siete socios, del consorcio formado para el desarrollo del proyecto Learning4Work $(L 4 W)$, enmarcado dentro del programa Erasmus+ de la Comunidad Europea. El objetivo del proyecto es constatar si las metodologías de aprendizaje activo aplicadas a entornos de Formación Profesional mejoran la motivación y rendimiento de los estudiantes y significan una mejora significativa en el mundo laboral. Los resultados preliminares nos muestran la necesidad de adaptar los contenidos de forma específica al ámbito de cada escuela para mejorar los resultados, ya que sin una fase de contextualización los estudiantes no acaban percibiendo la potencialidad del método.

\section{Palabras Clave}

Innovación Educativa; Metodologías de Aprendizaje; Formación Profesional; Perfil del usuario; Estudios de Usabilidad y Satisfacción: Aprendizaje Activo.

\begin{abstract}
This article presents the results and the degree of student satisfaction obtained in the usability testing of the Scenario Centered Curriculum (SCC) method, with a specific focus on the technological profile of each group of students. The method was incorporated into a Digital Marketing course which was taught in three Vocation Training Schools in Spain, Italy and France. The schools involved, together with seven other members, form part of a consortium created to promote the development of the Learning4Work Project $(\mathrm{L} 4 \mathrm{~W})$, as part of the Erasmus+ program of the European Community. The aim of this project is to verify whether the active learning methods applied to Vocational Training environments improve student motivation and performance and bring about significant improvement in the workplace. The preliminary results obtained indicate a need to adapt the contents to the specific professional area of each school in order to improve the results, as it appears that without a contextualization phase, students do not fully appreciate the potential advantages of the method
\end{abstract}

\section{Keywords}

Educational Innovation; Learning Methodologies; Vocational Training; User Profile; Satisfaction and Usability Studies; Active Learning. 


\section{Introducción}

El Proyecto Learning4Work está conformado por diez socios internacionales. La Salle Campus Barcelona - Universitat Ramon Llull (España) adquiere el rol de investigador principal. Tres socios más actúan como coordinadores de tareas: la Federazione Istituti Di Attività Educative (FIDAE) e Intesa SanPaolo Formazione en Italia y la Association Europeenne des Directeurs d'Institutions Lasalliennes (ASSEDIL) por Francia. Además de seis escuelas de Formación Profesional, dos por cada país, que forman parte del proyecto donde se desarrollarán los casos de estudio: Los colegios La Salle Palma y Salesians de Sarrià por España, el Instituto Cavanis y Suore Salesiane dei Sacri Couri por Italia y Ensemble Solaire Jeanne d'Arc y Groupe Scolaire Saint Joseph La Salle por Francia.

El proyecto tiene como objetivos principales reducir la tasa de abandono de los estudiantes de Formación Profesional (FP), así como reforzar el aprendizaje de forma más efectiva, perdurable y que pueda favorecer la inserción laboral de dichos estudiantes. Para ello, la propuesta rediseña los métodos de aprendizaje clásicos mediante la utilización de mundos inmersivos (Hew, Cheung, 2010) y el aprendizaje por roles y basado en proyectos, más conocido como Project Based Learning (Hmelo-Silver, 2004; Edens, 2000). En concreto se utilizará el método Scenario Centered Curriculum (Popkewitz, 1977; Schank, 2002) como estrategia de aprendizaje activo. Esta metodología se ha utilizado previamente con éxito, destacando por su impacto o cercanía al grupo de trabajo de la Carnegie Mellon (Koopman, et al., 2005; Bareiss \& Griss, 2008) o en la Universitat Oberta La Salle (Higueras, 2014).

Como objetivos secundarios se establecen el desarrollo de competencias mediante el trabajo cooperativo en entornos internacionales, ampliar el uso de herramientas y sistemas TIC (Tecnologías de la Información y la Comunicación) y generar un marco de trabajo fácilmente extrapolable a otros ámbitos de estudio y para todo tipo de escuelas. Dada la proximidad de los estudiantes de FP con el mundo laboral se antoja básico mejorar cualquier actividad formativa con el fin de:

- Generar metodologías de aprendizaje motivadoras, efectivas, internacionales y prácticas para estudiantes de FP de los países que componen el consorcio, prestando especial atención al perfil del estudiante, ya que tanto su nivel tecnológico como académico pueden influir de forma directa en su grado de empleabilidad final en relación con las metodologías propuestas.

- Modificar y tener disponibles programas educativos basados en entornos inmersivos, proyectos y roles que se asemejen a tareas reales del mundo laboral.

- Fomentar el trabajo cooperativo en entornos internacionales y con estudiantes heterogéneos de 
forma que se pueda discernir en qué grado se pueden aplicar lineal o simultáneamente diferentes métodos educativos innovadores y verificar finalmente si estos métodos mejoran la forma de hacer de los tradicionales centros de FP.

El presente artículo se ha focalizado en analizar la relación existente entre el perfil tecnológico de los estudiantes que han desarrollado por primera vez un curso de Marketing Digital mediante el método SCC y la percepción de los mismos en cuanto a la usabilidad del método y el grado de satisfacción con el curso. Los datos que se presentan y analizan provienen de las tres escuelas, una por cada país del consorcio (España, Italia y Francia), que han realizado el curso de Marketing Digital (MD) mediante SCC. En paralelo y como posteriormente se observa en la descripción de las fases del proyecto, las otras tres escuelas han realizado el curso de MD mediante metodología tradicional. En una fase posterior del Proyecto L4W se incorporará la metodología SCC en todas las escuelas participantes con la impartición de otro curso creando un aprendizaje colaborativo con grupos de estudiantes internacionales.

Como se verá en el análisis de los datos y en las posteriores conclusiones, se han detectado diversos factores que han influido de forma determinante en los resultados. Entre estos factores no contemplados previamente, pero sí referenciados en la bibliografía sobre innovación docente y tecnología (Damanpour, 1987; King, et al., 1994; Romiszowski, 2004; Law, Pelgrum \& Plomp, 2008), se pueden destacar: problemas burocráticos de las direcciones de las escuelas para incorporar los cursos en su plan docente, falta de una estrategia de integración adecuada por los docentes en el horario académico de los cursos y una desmotivación inicial de los alumnos al no entender la necesidad del curso ofrecido. Estos aspectos condicionan los resultados obtenidos y son un elemento clave a tener en consideración para las mejoras a introducir en los siguientes cursos a impartir.

\section{Marco teórico}

\subsection{Contexto de aprendizaje actual en FP}

La FP se considera actualmente un ámbito educativo en expansión dada su capacidad de preparar a sus estudiantes de cara a una salida laboral inminente (Endedijk, Vermunt, Meijer \& Brekelmans, 2014). Esta capacitación viene reforzada por unas demandas sociales de profesionales con habilidades y competencias muy específicas, capaces de resolver todo tipo de problemáticas especialmente desde un punto de vista tecnológico, colaborativo e innovador.

Por otro lado, hay que destacar cómo en toda la Unión Europea (UE) se ha cifrado en un 11,9\% el 
Abandono Escolar Prematuro (AEP) y, aunque dicha tasa se ha reducido en los últimos años (informe 2013 de la oficina estadística Eurostat, 11-04-2014), todavía está lejos del 10\% previsto como objetivo para el 2020. Dicha situación es más crítica en países como España, con un 23.5\%, seguida de Malta, Portugal, Rumanía e Italia (17\%) y lejos incluso de la zona media donde se sitúa Francia con un 9.7\%. En este sentido, se han definido una serie de prioridades estratégicas comunitarias para el periodo 2014-2020 (Böhme Doucet, Komornicki, Zaucha \& Świątek, 2011), con el fin de:

- Desarrollar las habilidades básicas y transversales, así como de las relacionadas con la capacidad emprendedora de los alumnos, sus habilidades digitales, y las capacidades de comunicación multi-idiomática en el ámbito de la juventud y la FP.

- Desarrollar sistemas adaptados de evaluación y certificación de los métodos propuestos basados en las salidas educacionales del ámbito estudiado.

- Intensificar y aumentar el uso de las TIC en los jóvenes para nuevos sistemas de aprendizaje no formal a través del soporte a nuevas actividades de aprendizaje mediante Open Educational Resources (Atkins, Brown \& Hammond, 2007; García-Peñalvo, García de Figuerola, \& Merlo-Vega, 2010; Ramírez-Montoya \& García-Peñalvo, 2015).

- Promover una mayor coherencia entre los diferentes sistemas evaluativos de diferentes países de la UE, así como nuevas herramientas que permitan fácilmente reconocer entre sistemas educativos diferenciados las habilidades y calificaciones obtenidas por los estudiantes.

- Transferir las estrategias de aprendizaje y métodos a los estudiantes de FP en la mejora de su motivación y completar su educación e inserción laboral.

Como se verá posteriormente, el enfoque propuesto del proyecto centrado en el aprendizaje mediante SCC, parte de la base experimental (Estruch \& Silva, 2006; Thistlethwait, et al., 2012) que demuestra la mejora en la satisfacción de los estudiantes utilizando métodos que les permiten afrontar situaciones reales de su futuro laboral. Adicionalmente, podemos afirmar que el trabajo en equipo genera un mayor interés y motivación en la realización de todo tipo de tareas, lo cual acaba influyendo en los resultados académicos, así como en su competitividad futura (Shepperd, 1993). Esta línea marco de actuación, nace como prioridad a mediados de la década de los 90 en el seno de la Comunidad Económica Europea. En 1995, en el libro blanco de la Comisión “Enseñanza y aprendizaje: hacia la sociedad del aprendizaje", se aboga por el desarrollo de estrategias comunes sobre la integración entre educación, formación y trabajo y posteriormente, en los consejos de Feira y Lisboa del 2000, se prioriza el desarrollo de los nuevos sistemas de educación y formación, ya como fase previa al programa Europa 2020. 
La estrategia Europa 2020 está destinada a garantizar los trabajos en el 75\% de las personas de edades comprendidas entre los 20 y 64 años, las inversiones públicas y privadas en investigación y desarrollo alrededor del 3\% del PIB, reducir las emisiones de gases de efecto invernadero en un $20 \%$, en comparación con los niveles de 1990, proporcionar las fuentes de energía renovables en el consumo final de energía en al menos un 20\%, reducir la tasa de deserción escolar a menos de un 10\% y garantizar que al menos el 40\% de los jóvenes obtengan un diploma o título universitario con el fin de disminuir los cerca de 20 millones de personas que están en riesgo de caer en la pobreza. En conclusión, parece meridianamente claro que la educación orientada al trabajo y la formación, según la perspectiva de aprendizaje de toda la vida declarado en Lisboa, desempeñan un papel fundamental y cómo una correcta distribución, capacitación y evaluación por competencias debe ayudar a alcanzar dichos objetivos.

\subsection{Learning4Work: descripción del proyecto}

Con el fin de abordar las deficiencias estructurales de los estudios de FP y mejorar las tasas de AEP, en el proyecto se presentan nuevos enfoques en los siguientes términos:

- Aplicar el método SCC, no experimentado previamente en los estudios de FP, como estrategia de aprendizaje activo.

- Evaluar inicialmente el riesgo de AEP para un posterior seguimiento del alumno mediante la motorización conjunta con la empresa (empleabilidad global del alumno a lo largo de todo el proyecto).

- Evaluar previamente los perfiles de usuario existentes y generar grupos piloto homogéneos para la impartición de un curso de Marketing Digital (MD), tanto desde un punto de vista tradicional como usando estrategias de aprendizaje colaborativo.

- Generar, en una segunda fase, un curso SCC centrado en Mobile Commerce (MC) e impartirlo en un entorno nacional y también en un entorno internacional, mediante prácticas colaborativas. Con esto los alumnos abordan problemáticas reales del mundo laboral centrados en el uso innovador de nuevas tecnologías. Adicionalmente este método, de forma intrínseca, les ayuda a mejorar sus competencias, tanto básicas como transversales, y también las específicas relacionadas con las competencias digitales básicas.

La evaluación del riesgo de AEP, la adopción de soluciones educativas innovadoras basadas en SCC y el trabajo colaborativo transnacional promueve una fuerte coherencia de los sistemas educativos de los diferentes países de la UE y facilita un mejor reconocimiento de las competencias adquiridas. 
El proyecto, por tanto, potencia tanto en contenido como en los métodos y herramientas, unos modelos innovadores de transferencia de acuerdo con las directrices sectoriales (tanto a nivel local, nacional como europeo), con el fin de integrar contenidos, métodos y resultados para su posterior transferencia educativa a otros centros, sistemas de educación y otros socios, tanto de los países del proyecto como de las redes educativas de los centros implicados.

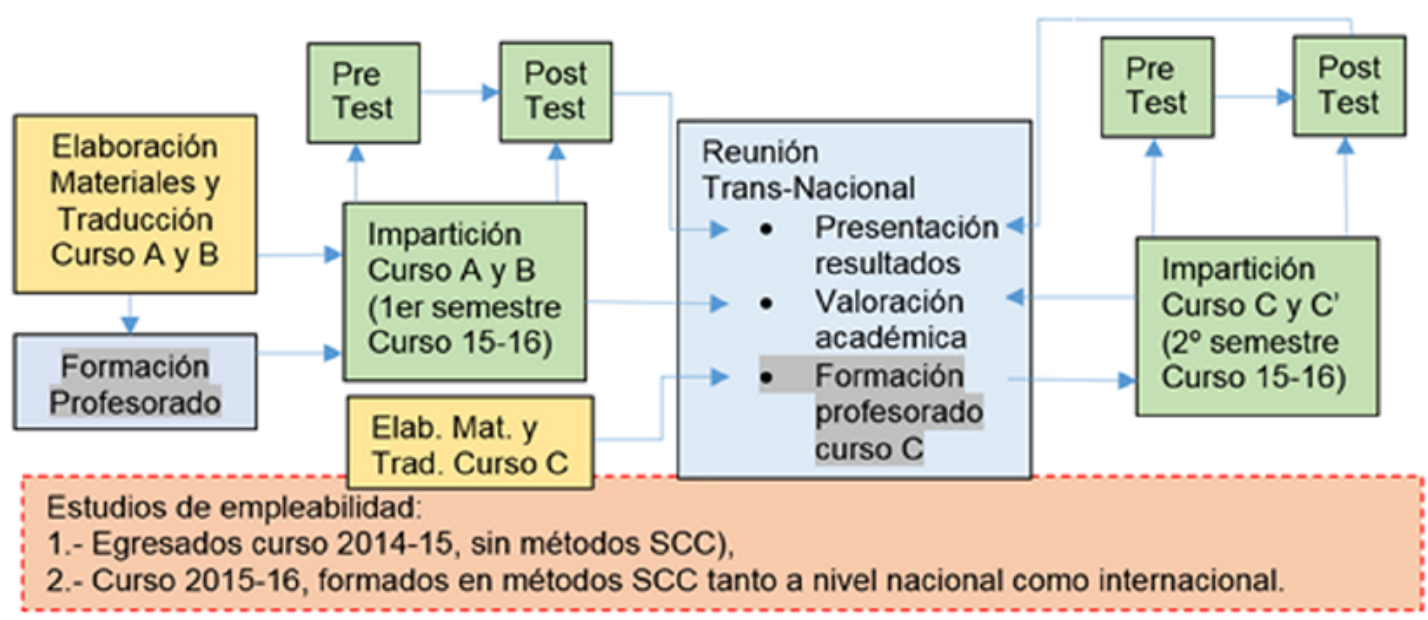

Figura 1. Diagrama global del proyecto. Curso A: MD Tradicional; curso B: MD SCC; curso C: MC SCC local, curso C': MC SCC Internacional.

El proyecto se divide en cinco fases generales no necesariamente secuenciales y que seguirían el esquema de trabajo que se observa en la Figura 1:

- Preparación. Básicamente se realizan tareas de gestión y coordinación entre los socios, habitualmente antes del inicio de los cursos y después de su finalización.

- Generación de contenidos. En esta fase se preparan diseñan y crean todos los contenidos relacionados con la ejecución del proyecto: materiales docentes del curso de Marketing Digital (MD) y del curso de Mobile Commerce (MC) mediante SCC, materiales docentes del curso MD mediante una metodología tradicional, materiales para la formación de los profesores sobre la metodología docente SCC y el modelo de recogida de datos para la elaboración de indicadores (académicos y empleabilidad). Como norma general todos los materiales se producen inicialmente en inglés y cada socio coordinador los traduce al idioma de su país.

- Implantación. En esta fase se forma al profesorado de las escuelas implicadas que posteriormente van a impartir los cursos. Antes del inicio de los cursos se realiza una evaluación inicial de los estudiantes (o Pre-Test para evaluar el perfil del alumno) y al finalizar el mismo una evaluación de las competencias adquiridas (o Post-Test, que también incluye la evaluación del grado de satisfacción de la experiencia).

- Seguimiento. En esta fase y, según el modelo de datos diseñado, se recoge la información del perfil del estudiante y se analiza el grado de inserción laboral, su relación con la metodología 
propuesta en el proyecto y la evolución de su perfil. Esta información se ha de contrastar con los indicadores de inserción laboral de los alumnos de cursos previos, a los cuales no se les ha aplicado dichas metodologías de aprendizaje.

- Análisis de resultados y difusión. Al final de cada una de los cursos, cada miembro coordinador realiza un análisis de los resultados obtenidos en los centros de su país y presenta sus conclusiones en un informe. El socio coordinador del proyecto elabora un análisis global con todos los resultados. Estos resultados se ponen en común en los dos workshops que se realizan al finalizar cada uno de los cursos.

\subsection{Descripción del método Scenario Centered Curriculum}

EI SCC está basado en el modelo Constructivista de cómo la gente aprende (Savery \& Duffy, 1995) y en el modelo de memoria dinámica sobre cómo la memoria humana se desarrolla (Schank, 1983). Estas teorías sugieren que la gente aprende de forma natural construyendo el nuevo conocimiento, añadiendo lo que individualmente ya saben y a partir de sus propias experiencias previas (Kolb, 2014). La implicación para la enseñanza deriva en que los nuevos entornos de aprendizaje deben situar a los alumnos en experiencias que desafiarán sus concepciones previas y permitirán que ellos comparen lo que experimentan con lo que antes creían: darse cuenta de las diferencias lleva a la curiosidad, aspecto clave que lleva al aprendizaje.

Existen dos métodos principales de la enseñanza en un entorno SCC. Uno basado en el aprendizaje cognitivo (Terenzini, Pascarella \& Blimling, 1996) y otro centrado en el método Socrático Interrogativo (Hintikka, 1993). El papel del profesor, que en SCC pasa a ser un mero mentor, en el primer método su trabajo se centra en mostrar modelos de trabajo similares a la problemática a resolver, apoyar a los alumnos reduciendo progresivamente dicho soporte y no deja de guiarlos en todo momento para generar una actitud de constante reflexión sobre el aprendizaje realizado y cómo este se puede llevar a otros contextos (Bareiss \& Griss, 2008). En el caso del método socrático, el procedimiento de enseñanza se basa en la pregunta constante, de forma que se mantiene una conversación constante entre profesor y alumnos a base que estos respondan y saquen conclusiones de las preguntas de partida que el tutor realiza. A partir de las preguntas realizadas por los alumnos el mentor no da directamente la respuesta o solución, ya que este orienta adecuadamente al alumno mediante otra pregunta o reflexión sobre cómo el alumno puede encontrar la respuesta a su duda, actuando el profesor como mentor o facilitador de la solución. Esto facilita que sea el mismo alumno el que encuentre la respuesta a su duda o pregunta.

EI SCC coloca a los estudiantes en un contexto realista y pide que ellos hagan tareas significativas 
dentro de ese contexto antes de que reciban cualquier tipo de formación. Tienen la oportunidad de aplicar las intuiciones que tengan que ver con el problema, tanto para avanzar sobre ellas, como para realizarse preguntas antes de empezar a trabajar. Este método permite aprender en el mismo momento en que lo necesitan y que los alumnos sientan curiosidad, a la par que también les permite ir cambiando sus intuiciones (o la falta de las mismas). Esta curiosidad viene motivada por la evidencia del alumno de que no sabe resolver el problema, lo que conlleva a su interés en encontrar la solución, facilitando todo este proceso su adquisición de los nuevos conocimientos.

En esta metodología de trabajo, los profesores adoptan diferentes tipos de roles en el desarrollo del curso. A veces los roles no se distinguen claramente en una sola sesión con los estudiantes y se van definiendo a medida que los alumnos avanzan con la realización del proyecto. Los roles principales del profesor son: instructor o profesor, entrenador o coach y realizador de un rol específico.

En el rol de profesor se asume en un contexto formal sin caer en modelos convencionales de enseñanza. El profesor se sitúa como un facilitador en momentos puntuales del trabajo: al inicio y final del programa, en momentos evaluativos, liderando reuniones de trabajo y en general proporcionando informes de la evolución del trabajo.

El rol de coach es el papel más estrechamente vinculado con el modelo "mentor-aprendiz". El coach ayuda a los estudiantes y los grupos de estudiantes con una relación más íntima que en las reuniones semanales. El coach debe asegurarse de que todos los miembros están siguiendo el programa, ayuda con las estrategias de trabajo, configurando los equipos, ayudando a los grupos con ideas clave, identifica, evalua y aconseja sobre los trabajos personales de cada miembro del equipo, etc.

Finalmente, la tarea del profesor como realizador de un rol es necesaria cuando los alumnos tienen que interactuar con un perfil de usuario que no está directamente asignado, como por ejemplo podría ser un cliente, el jefe de la empresa, etc. Idealmente no obstante es mejor que esta tarea la ejecute otro profesor antes que el mentor del grupo.

A partir de todo ello, podemos intuir que la planificación y estructura de implantación de un curso SCC no es la convencional. Existe una estructura recomendada para las reuniones regulares con los estudiantes. Dicha estructura está diseñada para proporcionar a los estudiantes un nivel suficiente de apoyo y asistencia. Sin embargo, los profesores deben ajustar la estructura recomendada para adaptarse a las necesidades del curso y el conjunto de estudiantes. Una vez detallado el proceso de aprendizaje, funciones de los participantes y planificación de la enseñanza, se describe el modelo utilizado para la evaluación de los estudiantes, ya que este difiere del utilizado en procesos de aprendizaje convencionales. Se distinguen tres aspectos diferenciales.

- La metodología SCC se centra en la práctica de las competencias y en la utilización de los 
conocimientos necesarios que se requieren para la realización de una tarea que está relacionada con los objetivos del curso o programa, de forma contraria a la valoración del estudiante mediante la memorización o comprensión de un concepto.

- El trabajo y el desarrollo de los entregables se realiza en grupo y no de forma individual.

- Independientemente de la valoración y evaluación del profesor, los estudiantes puntúan entre ellos el trabajo realizado y su aportación a los trabajos y procesos realizados en grupo.

\section{Caso de estudio}

El curso propuesto posiciona a los alumnos como miembros del departamento de marketing online de una consultoría cualquiera. El objetivo primario de dicho departamento es aumentar la visibilidad en Internet de sus clientes. El caso de estudio se centra en posicionar una empresa de videojuegos exclusivamente para Internet. Los tipos de juegos propuestos son altamente innovadores, ya que además de trabajar con tecnología 3D pretenden que sea multi-soporte y adaptable a cualquier navegador, red social o portal de aplicaciones para los principales sistemas operativos de dispositivos móviles. Los alumnos deben trabajar en diversas líneas de acción:

- Analizando el posicionamiento actual de la empresa.

- Proponiendo cómo debería ser la web del cliente para aumentar la captación de clientes.

- Analizar las herramientas actuales de la red que mejor encajarían en la estrategia de expansión de la empresa.

- Generar una estrategia de posicionamiento a 12 meses que, minimizando los gastos, maximice el posicionamiento del cliente y el impacto de sus nuevas propuestas.

Como resultado de su trabajo los alumnos desarrollan y presentan los entregables siguientes:

1. Un informe de Análisis SEO (Search Engine Optimization).

2. Un informe de Análisis SEM (Search Engine Marketing).

3. Un informe de Posicionamiento SMM (Social Media Marketing).

4. Una Presentación final donde se desarrolla y presenta la solución propuesta al cliente final.

Centrados en el enfoque del presente artículo, y sin olvidar los objetivos globales del proyecto, un aspecto fundamental a tratar es el seguimiento y evaluación del alumno. Para ello se han definido 
una serie de test a realizar a lo largo de todo el proyecto que se ajustarían a la siguiente planificación (ver Tabla 1).

\begin{tabular}{|c|c|c|c|c|}
\hline Curso & Tipo & Evaluación & Cuándo & Método Análisis \\
\hline \multirow[b]{2}{*}{$\stackrel{\rho}{\Sigma}$} & $\begin{array}{c}\text { Ambos } \\
(1)\end{array}$ & $\begin{array}{l}\text { 1.- Perfil tecnológico } \\
\text { 2.- Motivación (2) }\end{array}$ & $\begin{array}{l}\text { Antes de } \\
\text { empezar }\end{array}$ & $\begin{array}{c}\text { Encuesta cuantitativa tipo } \\
\text { Likert }\end{array}$ \\
\hline & $\begin{array}{c}\text { Ambos } \\
(1)\end{array}$ & $\begin{array}{l}\text { 3.- Habilidades generales } \\
\text { 4.- Habilidades específicas } \\
\text { 5.- Usabilidad del método } \\
\text { 6.- Satisfacción del estudiante }\end{array}$ & $\begin{array}{l}\text { Al final del } \\
\text { curso }\end{array}$ & $\begin{array}{c}\text { Método mixto: encuesta } \\
\text { cuantitativa y entrevista } \\
\text { cualitativa }\end{array}$ \\
\hline \multirow[b]{2}{*}{$\stackrel{\cup}{\Sigma}$} & $\begin{array}{l}\text { SCC } \\
(3)\end{array}$ & $\begin{array}{l}\text { 1.- Perfil tecnológico } \\
\text { 2.- Motivación }\end{array}$ & $\begin{array}{l}\text { Antes de } \\
\text { empezar }\end{array}$ & $\begin{array}{c}\text { Encuesta cuantitativa tipo } \\
\text { Likert }\end{array}$ \\
\hline & $\begin{array}{l}\text { SCC } \\
(3)\end{array}$ & $\begin{array}{l}\text { 3.- Habilidades generales } \\
\text { 4.- Habilidades específicas } \\
\text { 5.- Usabilidad del método } \\
\text { 6.- Satisfacción del estudiante } \\
\text { 7.- Impacto Internacional } \\
\text { 8.- Eficiencia del método SCC }\end{array}$ & $\begin{array}{l}\text { Al final del } \\
\text { curso }\end{array}$ & $\begin{array}{c}\text { Método mixto: encuesta } \\
\text { cuantitativa y entrevista } \\
\text { cualitativa }\end{array}$ \\
\hline
\end{tabular}

Tabla 1. Esquema de las evaluaciones. (1) Para MD-Tradicional y SCC, (2) Solo para grupo SCC, (3) Para ambos grupos, nacional e internacional.

Tal y como hemos definido previamente, el presente trabajo aborda el diseño y análisis preliminar de los resultados obtenidos del pre-test de perfil tecnológico del alumno y de los post-test correspondientes a usabilidad del método y satisfacción del estudiante, todos ellos centrados en el curso de MD, único por el momento con datos procesados. En el momento de escritura del presente artículo se están procesando el resto de datos correspondientes al curso de MD, tanto del resto de test, como de los resultados académicos de ambos grupos, los que han desarrollado el sistema en método tradicional, así como aquellos grupos que han utilizado SCC.

\subsection{Pre-Test: Perfil Tecnológico del estudiante}

La información inicial que aporta la realización de un pre-test es de vital importancia de cara al posterior análisis de los datos del post-test. Por un lado debe servir para evaluar el grado de diferencia existente entre los grupos de trabajo y de esta forma discutir si los resultados del trabajo se deben a la aplicación de una metodología de trabajo nueva o por el contrario simplemente a que los grupos en sí mismos son diferentes y generan respuestas diferenciadas. Así mismo, y en el caso del test de perfil tecnológico, va a permitir caracterizar las muestras de trabajo y evaluar de forma preliminar si la implantación de un sistema de trabajo nuevo se adaptará o no al perfil de estudiantes. El test diseñado para evaluar el perfil de los estudiantes del proyecto se puede ver en la Tabla 2. 


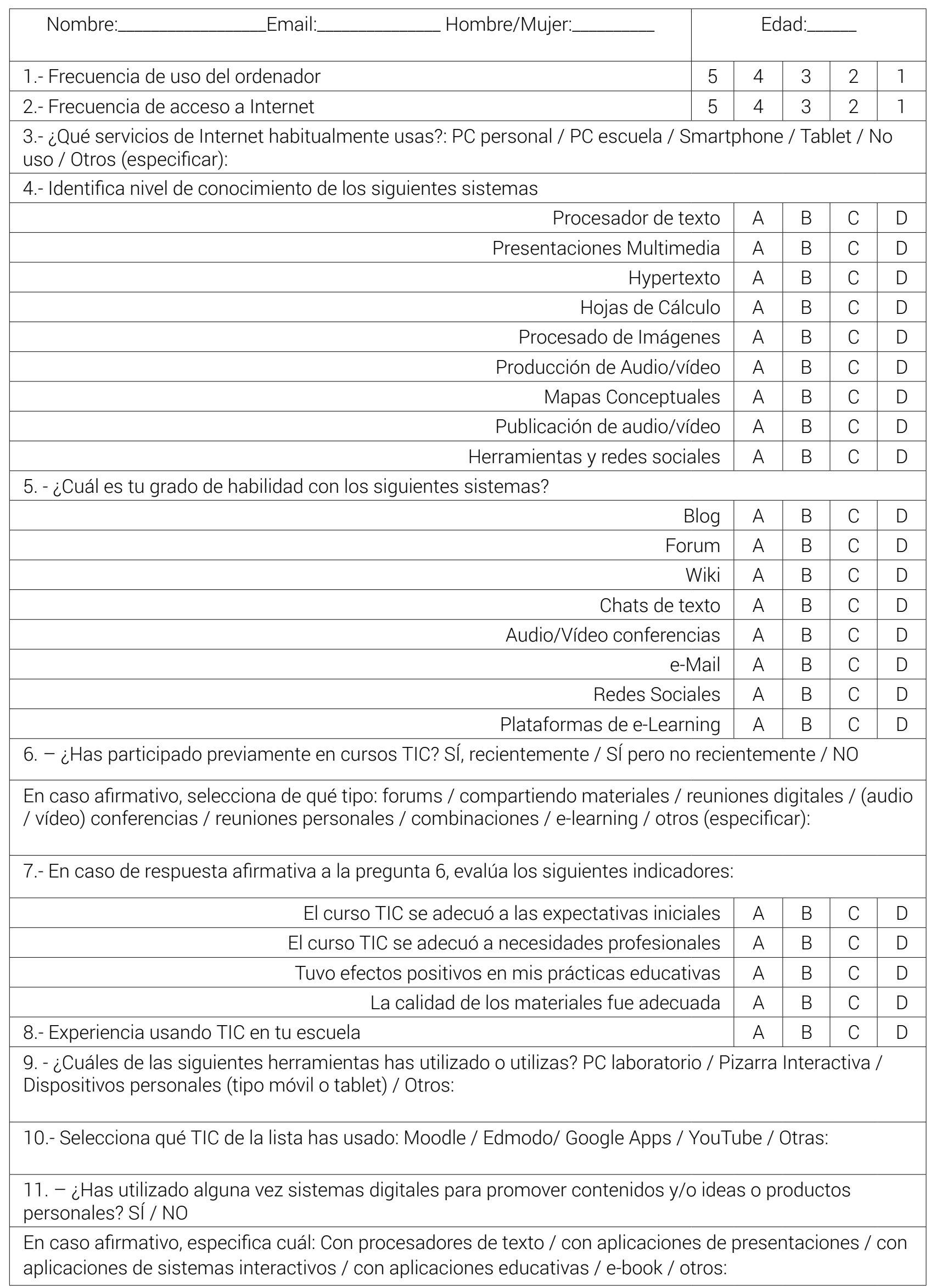

Tabla 2. Pre-Test. Perfil tecnológico del alumno. Preguntas 1 y 2: escala de 1-Nunca a 5-Diariamente. Preguntas 4, 5 y 8: escala de A-Mucho a D-Nada. 


\subsection{Post-Test: Usabilidad del método SCC y grado de satisfacción del alumno}

Tal y como se comenta en la Tabla 1, el post test de los dos cursos se basa en un total de seis encuestas. En ellas se evalúan tanto la adquisición de competencias generales y específicas como los conceptos de usabilidad, satisfacción, interacción internacional, etc. Estas encuestas, junto con los resultados académicos de cada curso, conformarán el global de resultados a analizar para confirmar si las innovaciones propuestas surgen para empezar un impacto positivo en el alumno y en el aula en general. En una fase futura, se tendrá que analizar si esta formación ha servido a los actuales alumnos para una más rápida, efectiva y satisfactoria incorporación al mundo laboral.

Los test que en el presente trabajo se analizarán respecto al perfil previo de los alumnos son el de usabilidad del método SCC (ver Tabla 3) y el de satisfacción del alumno (ver Tabla 4).

\begin{tabular}{|c|c|c|c|c|}
\hline $\begin{array}{l}\text { Nombre:_Email:__ Mujer/ } \\
\text { Hombre:_ }\end{array}$ & Edad: & & & \\
\hline PREGUNTA & Mucho & Suficiente & Poco & Nada \\
\hline 1.- El método de formación SCC es accesible & A & B & C & $\mathrm{D}$ \\
\hline $\begin{array}{l}\text { 2.- El contenido, estilo, equipo y material didáctico } \\
\text { de enseñanza es adecuado a los objetivos }\end{array}$ & A & B & C & $\mathrm{D}$ \\
\hline $\begin{array}{l}\text { 3.- El tiempo dedicado a la aplicación de la metod- } \\
\text { ología de SCC en la planificación escolar es adecua- } \\
\text { do }\end{array}$ & A & B & C & D \\
\hline $\begin{array}{l}\text { 4.- El estilo de conducción de la formación centrada } \\
\text { en la SCC se caracterizó por el dominio del conteni- } \\
\text { do y la claridad }\end{array}$ & A & B & C & $\mathrm{D}$ \\
\hline 5.- Aula y apoyos didácticos en línea son adecuados & A & B & C & $\mathrm{D}$ \\
\hline $\begin{array}{l}\text { 6.- SCC fomenta la colaboración y trabajo en equipo } \\
\text { para realizar tareas y alcanzar objetivos }\end{array}$ & A & B & C & D \\
\hline $\begin{array}{l}\text { 7.- La cantidad de materiales de enseñanza ha sido } \\
\text { adecuada }\end{array}$ & A & B & C & $\mathrm{D}$ \\
\hline $\begin{array}{l}\text { 8.- Los materiales estaban completos y fáciles de } \\
\text { usar }\end{array}$ & A & B & C & $\mathrm{D}$ \\
\hline $\begin{array}{l}\text { 9.- El soporte tecnológico y el equipo fueron } \\
\text { intuitivos y fáciles de usar, adecuada y } \\
\text { funcionalmente }\end{array}$ & A & B & C & D \\
\hline
\end{tabular}




\begin{tabular}{|c|c|c|c|c|}
\hline $\begin{array}{l}\text { Nombre:_Email:_______ } \\
\text { Mujer/Hombre:_ }\end{array}$ & \multicolumn{4}{|c|}{ Edad:___ } \\
\hline \multicolumn{3}{|l|}{ 1.- Los contenidos del curso están claros } & sí & NO \\
\hline \multicolumn{3}{|c|}{$\begin{array}{l}\text { 2.- El método de implementación del curso ha sido novedoso respecto al sistema en } \\
\text { que habitualmente realizas los cursos }\end{array}$} & Sí & NO \\
\hline \multicolumn{5}{|c|}{ 2.1.- Si la anterior respuestas es afirmativa, ¿de qué forma? (indicar solo una opción) } \\
\hline \multicolumn{5}{|l|}{ Han estimulado más mi participación } \\
\hline \multicolumn{5}{|c|}{ Me han permitido experimentar y poner en práctica el conocimiento adquirido } \\
\hline \multicolumn{5}{|l|}{ Me han hecho pensar más acerca de los contenidos } \\
\hline \multicolumn{5}{|l|}{ Me han permitido relacionar los contenidos con la vida real } \\
\hline \multicolumn{5}{|l|}{ Otros (detallar): } \\
\hline 3.- Nivel de satisfacción respecto de: & Mucho & Sufici. & Poco & Nada \\
\hline 3.1.- Relevancia del material distribuido & A & B & C & D \\
\hline 3.2.- Claridad y abarque de las presentaciones & A & B & C & D \\
\hline 3.3.- Comunicación efectiva & A & B & C & D \\
\hline 3.4.- Duración de la presentaciones adecuada & A & B & C & D \\
\hline 3.5.- Utilidad y concreción de la información dada & A & B & C & $\mathrm{D}$ \\
\hline 3.6.- Docencia clara & A & B & $\mathrm{C}$ & $\mathrm{D}$ \\
\hline 3.7.- Parte práctica y ejercicios adecuados & A & B & C & $\mathrm{D}$ \\
\hline 3.8.- Consistencia entre parte teórica y práctica & A & B & C & $\mathrm{D}$ \\
\hline 3.9.- Utilidad del conocimiento adquirido en la vida práctica & A & B & C & D \\
\hline 3.10.- Desarrollo de la creatividad & A & B & C & $\mathrm{D}$ \\
\hline 3.11.- Acceso a las tecnologías & A & B & $\mathrm{C}$ & $\mathrm{D}$ \\
\hline \multicolumn{5}{|c|}{$\begin{array}{l}\text { 4.- De acuerdo con lo indicado en el apartado anterior, globalmente me considero: SATISFECHO / NO } \\
\text { SATISFECHO }\end{array}$} \\
\hline \multicolumn{5}{|c|}{$\begin{array}{l}\text { 5.- ¿Cuál de los siguientes aspectos sugiere mejorar o cambiar para que el curso a su vez pueda mejorar? } \\
\text { (Indicar con una X todos aquellos aspectos que necesitan mejorar): }\end{array}$} \\
\hline \multicolumn{5}{|l|}{ 5.1.- Relevancia del material distribuido } \\
\hline \multicolumn{5}{|l|}{ 5.2.- Claridad y abarque de las presentaciones } \\
\hline \multicolumn{5}{|l|}{ 5.3.- Comunicación efectiva } \\
\hline \multicolumn{5}{|l|}{ 5.4.- Duración de la presentaciones adecuada } \\
\hline \multicolumn{5}{|l|}{ 5.5.- Utilidad y concreción de la información dada } \\
\hline \multicolumn{5}{|l|}{ 5.6.- Docencia clara } \\
\hline \multicolumn{5}{|l|}{ 5.7.- Parte práctica y ejercicios adecuados } \\
\hline \multicolumn{5}{|l|}{ 5.8.- Consistencia entre parte teórica y práctica } \\
\hline \multicolumn{5}{|l|}{ 5.9.- Utilidad del conocimiento adquirido en la vida práctica } \\
\hline \multicolumn{5}{|l|}{ 5.10.- Desarrollo de la creatividad } \\
\hline 5.11.- Acceso a las tecnologías & & & & \\
\hline
\end{tabular}




\section{Resultados}

El proyecto Learning4Work se inició a principios del año 2015 con la preparación de todos los materiales para los alumnos, materiales de soporte para los profesores de las escuelas, así como su posterior formación en los mismos centros escolares de España, Italia y Francia. El primer curso de formación (Marketing Digital) a los alumnos tuvo lugar a partir del mes de septiembre del 2015 y finalizó a principios del año 2016. La distribución de la formación en los diferentes países se puede ver en la Tabla 5.

\begin{tabular}{|c|c|c|c|c|c|c|}
\hline & \multicolumn{2}{|c|}{ SCC-ESP } & \multicolumn{2}{c|}{ SCC-ITA } & \multicolumn{2}{c|}{ SCC-FRA } \\
\hline & n. & $\%$ & n. & $\%$ & n. & $\%$ \\
\hline Hombres & 27 & 96,4 & 15 & 75,0 & 11 & 78,6 \\
\hline Mujeres & 1 & 3.6 & 5 & 25,0 & 3 & 21,4 \\
\hline
\end{tabular}

Tabla 5. Información de la muestra

Como se ha indicado previamente, antes de empezar la formación los estudiantes han realizado cuatro encuestas agrupadas, en lo que se ha denominado Pre-Test (Ver Tabla 1). Dentro de estas encuestas y para este caso, se focalizará el estudio en los principales datos obtenidos asociados con el perfil tecnológico de los alumnos y que en detalle se pueden observar en la Tabla 2. Los resultados principales se pueden ver en las siguientes Figuras 2, 3 y 4, donde se comparan los valores medios de cada país.

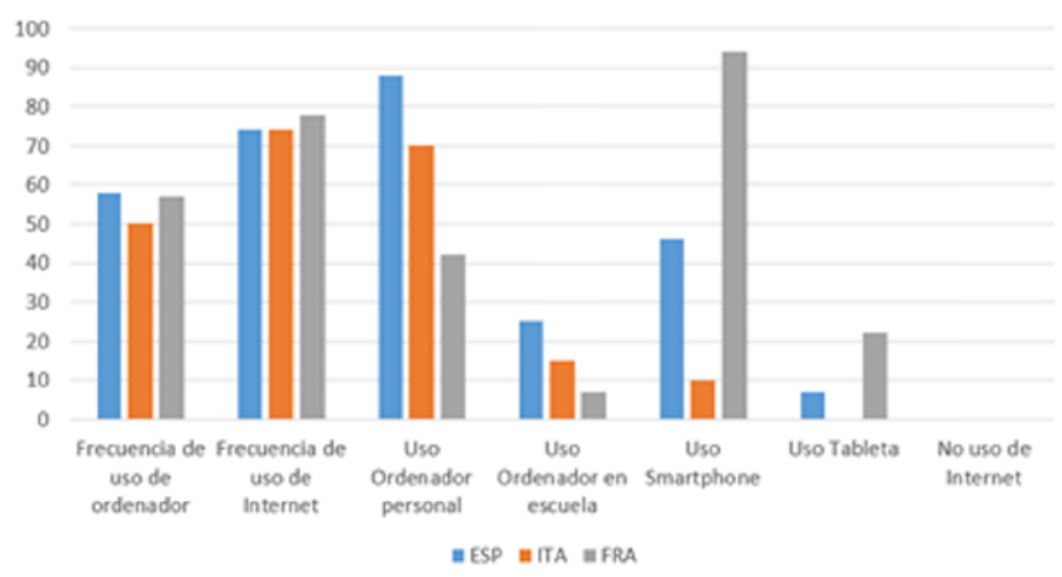

Figura 2. Pre-Test. Usos y frecuencia de trabajo con los dispositivos a utilizar en la experiencia (en porcentaje de uso y frecuencia diaría).

Inicialmente y como posteriormente se discutirá, ya se observa un comportamiento bastante heterogéneo entre los grupos de trabajo. Mientras las frecuencias de uso de ordenadores y conexiones a Internet es bastante pareja, no sucede lo mismo en referencia al tipo de tecnología que los estudiantes utilizan con mayor asiduidad. Mientras que en España e Italia se observa un uso mayoritario del ordenador personal, en Francia es donde se utilizan más los dispositivos móviles con funciones avanzadas como smartphones y tabletas. 
Pasando a evaluar la experiencia de uso de los alumnos con todo tipo de sistemas digitales, se puede ver un resumen de los valores en la Figura 3.

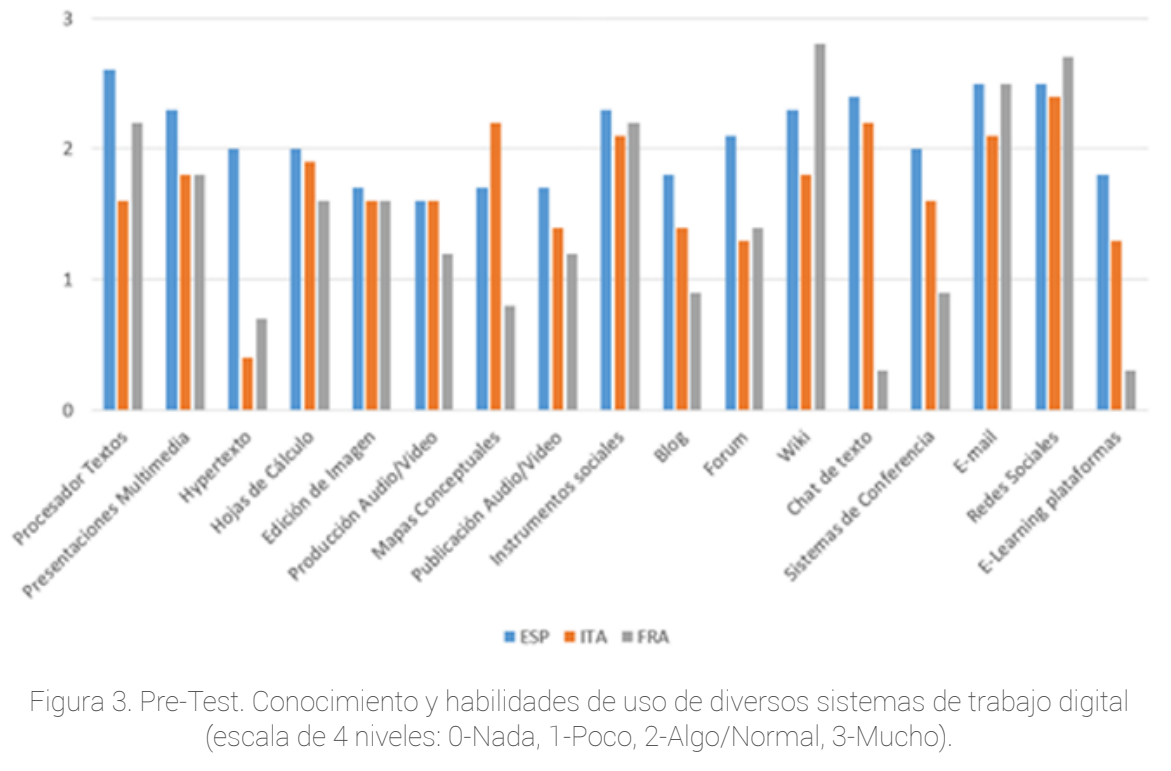

Como se puede observar, en este bloque de preguntas se detecta una clara diferencia entre los perfiles de los estudiantes. Globalmente, el grupo español está más acostumbrado al uso de las tecnologías sobre las cuales les hemos preguntado. Con un promedio general de 2.07 sobre 3 (y una desviación típica de 0.32), el grupo español demuestra un conocimiento mayor respecto al grupo italiano (con un promedio de 1.68 y una desviación de 0.47) y al grupo francés (con promedio de 1.47 y desviación de 0.79). Estadísticamente estas diferencias son significativas, ya que se sitúan por debajo del límite de 0.05 de significación (0.009 tanto para la comparativa entre ESP-ITA, como para ESP-FRA, mediante el análisis de los resultados comparando sus varianzas con la t-Student). Por el contrario, entre los grupos de Italia y Francia, no se puede concluir una diferencia significativa (al obtener la comparativa una significación de 0.3545 ), aspecto que indica que su media de conocimientos sobre tecnología no se puede considerar diferente.

Por último se analizan, dentro del test de perfil tecnológico de los alumnos, el resultado de usabilidad/ satisfacción de aquellos alumnos que han cursado previamente formaciones tecnológicas. Como se puede observar en la Figura 4, en general la valoración es muy baja para todos los alumnos en general, no llegando en ningún caso y para ninguna de las variables estudiadas ni siquiera al aprobado.

Valorando de forma global los resultados obtenidos del perfil tecnológico de los alumnos que realizarán la formación mediante el método SCC, se puede concluir que:

- Los cursos previos sobre temáticas TIC cursados por los alumnos no superan la puntuación de 1 sobre 3 en ningún caso, ya sea por promedio de cada variable por país $(0.67,0.91$, y 0.36 respectivamente, teniendo en cuenta que se sigue siempre el mismo orden de resultados españoles, italianos y franceses), o por índice encuestado $(0.56,0.65,0.60,0.78$ de cada variable de la Figura 4). 
- Los alumnos españoles tienen un perfil de uso de aplicaciones y tecnologías más elevado que sus colegas europeos, pero especialmente cuando estas se refieren al uso de ordenadores.

- Se puede destacar especialmente el uso de dispositivos móviles por parte de los estudiantes franceses, en un promedio muy superior a españoles e italianos, pero muy por debajo en cuanto a conocimientos en general sobre todo tipo de aplicaciones TIC y con la peor respuesta en formación previa de este tipo de tecnologías.

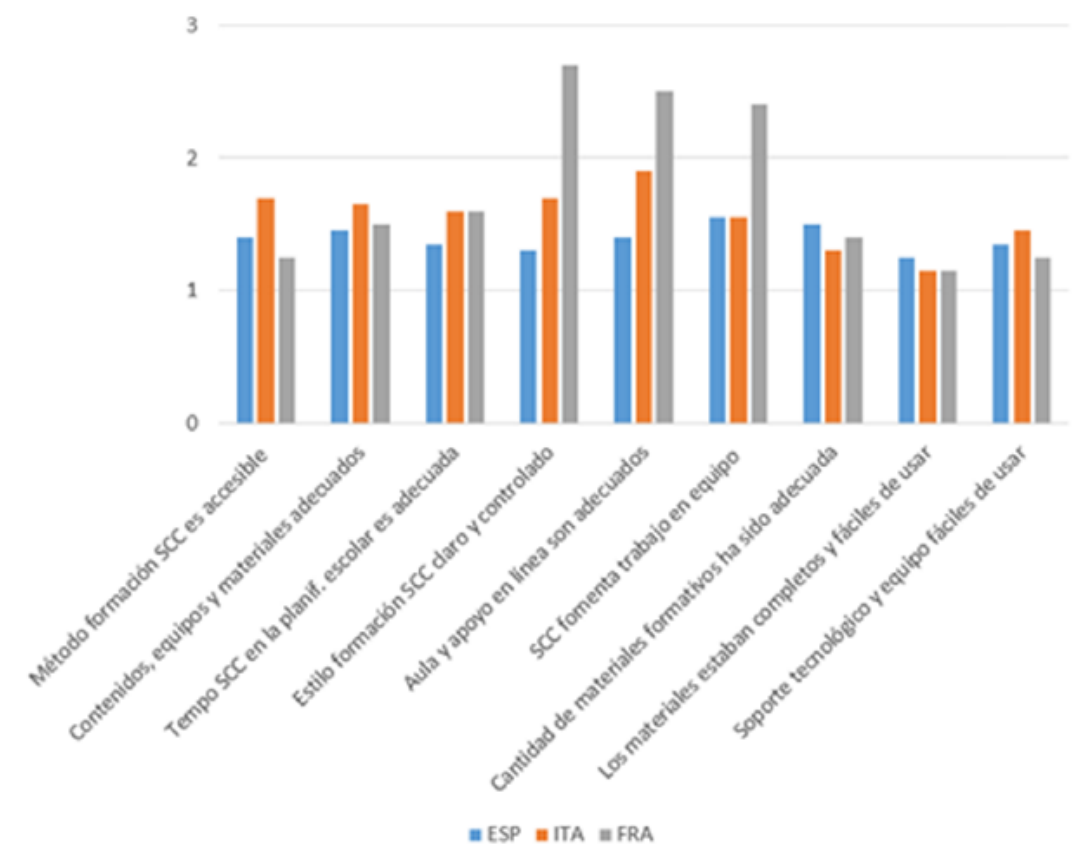

Figura 4. Pre-Test. Valoración de usabilidad de formación previa en TIC para aquellos alumnos que la han recibido dentro de los grupos de trabajo del proyecto con SCC.

A partir de los resultados obtenidos, y una vez realizado el curso de MD mediante SCC, los alumnos realizaron el Post-Test (Tabla 1), dentro del cual estaban definidas las encuestas de usabilidad y satisfacción según el diseño que podemos ver en las Tablas 3 y 4.

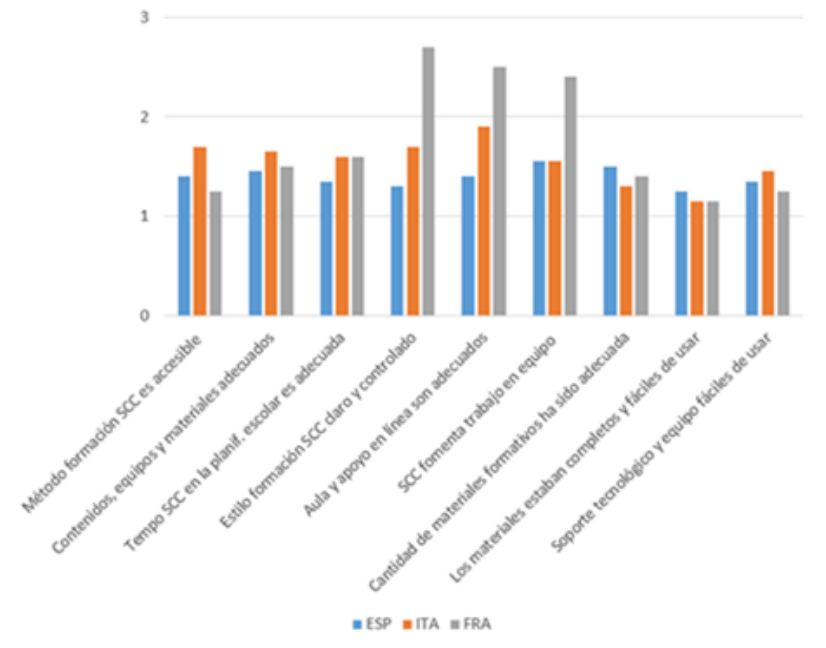

Figura 5. Post-Test. Usabilidad del método SCC. 
Analizando de forma global los resultados (Figura 5), el curso obtiene un promedio de 1.39 sobre 3 para el caso del grupo español (con una desviación de 0.09), un 1.55 para el italiano (desviación de 0.22) y un 1.75 para el grupo francés (con una desviación de 0.61). La diferencia entre los promedios y en especial en su desviación viene claramente definida por las respuestas a las preguntas relacionadas con el estilo de formación del SCC, el aula y el apoyo del curso y el trabajo en equipo que este sistema fomenta. Como queda claro, el grupo español (con un perfil tecnológico más elevado), es el que ha considerado que la facilidad de uso y trabajo con la metodología no ha sido fácil, y aunque todos los valores son bastante homogéneos, en la parte más negativa sitúa el estilo de formación y la usabilidad de los contenidos.

Con un perfil tecnológico medio, pero con unos mejores (aunque mínimos) antecedentes de formación TIC, el grupo italiano en general aprueba la usabilidad del curso, focalizando como aspectos negativos la cantidad de materiales disponibles para su seguimiento, así como al igual que para el grupo español, la usabilidad de los mismos. Con la peor respuesta a formaciones previas en TIC, y la menor experiencia en el uso de todo tipo de aplicaciones digitales, el grupo de estudiantes franceses es el que ha valorado con mejores puntuaciones la usabilidad del método SCC, valorando especialmente de forma positiva el estilo de formación, la disposición digital del aula y la ayuda en línea, así como el espíritu de fomento de trabajo en grupo que el SCC aporta a una temática. Al igual que para el resto de grupos, la peor valoración se la lleva la usabilidad de los materiales, indicador con un promedio global de 1.18 sobre 3, y como queda claro, es uno de los objetivos de mejora en el segundo curso a implementar a lo largo del segundo semestre del curso 2015-16.

Pasando a valorar el nivel de satisfacción de los estudiantes con el curso y la metodología propuesta, nos volvemos a encontrar con resultados bastantes heterogéneos. Gracias al test diseñado (ver Tabla 4), el cual evalúa diversos aspectos con diferentes enfoques, se pueden destacar los siguientes resultados:

- Solo el30,3\% de los estudiantes españoles se consideran globalmente satisfechos por la formación recibida. Este porcentaje aumenta hasta el $64.7 \%$ para el caso de los estudiantes italianos y se sitúa en una posición intermedia para el grupo francés con un $44.4 \%$.

- De forma global, el $47 \%$ de los estudiantes han considerado los contenidos claros, y un $62 \%$ de los alumnos perciben el método de trabajo como novedoso respecto al sistema clásico en que se plantean sus materias actuales. Los aspectos más destacados han sido la capacidad del método en hacer pensar al alumno más sobre los contenidos y la estimulación de la participación proactiva de los alumnos. 
- Centrados en el tercer apartado del test, las valoraciones globales por cada grupo respectivamente han sido de 1.34 (desviación de 0.10), 1.62 (desviación de 0.12) y 1.36 (desviación de 0.46), destacando, al igual que para el estudio de usabilidad, así como para los estudios de perfil de usuario, una gran desviación del grupo francés, aspecto a tener en cuenta en la valoración de sus resultados (ver Figura 6).

- Los mejores aspectos valorados en general por los alumnos han sido el acceso a las tecnologías (con un promedio de 1.85) y la docencia clara realizada (1.74, siempre sobre un máximo de 3 puntos). El resto de indicadores quedarían por debajo del umbral del aprobado, aspecto que nos indica la poca adaptación del curso en global a las expectativas/necesidades de los alumnos. Analizado los promedios de cada variable, podemos observar una diferencia significativa entre los promedios de la escuela española y la italiana (desviación o $p=0.00001$ ), mientras que no se puede corroborar dicha diferencia en el caso de comparar entre la escuela italiana y francesa, o la española y francesa. Al respecto, la elevada desviación de los resultados franceses en determinadas variables son factores que se cree que pueden influir en la comparativa de los resultados y que deben ser corroborados con los que se obtengan del segundo curso de Mobile Commerce, que actualmente se está impartiendo.

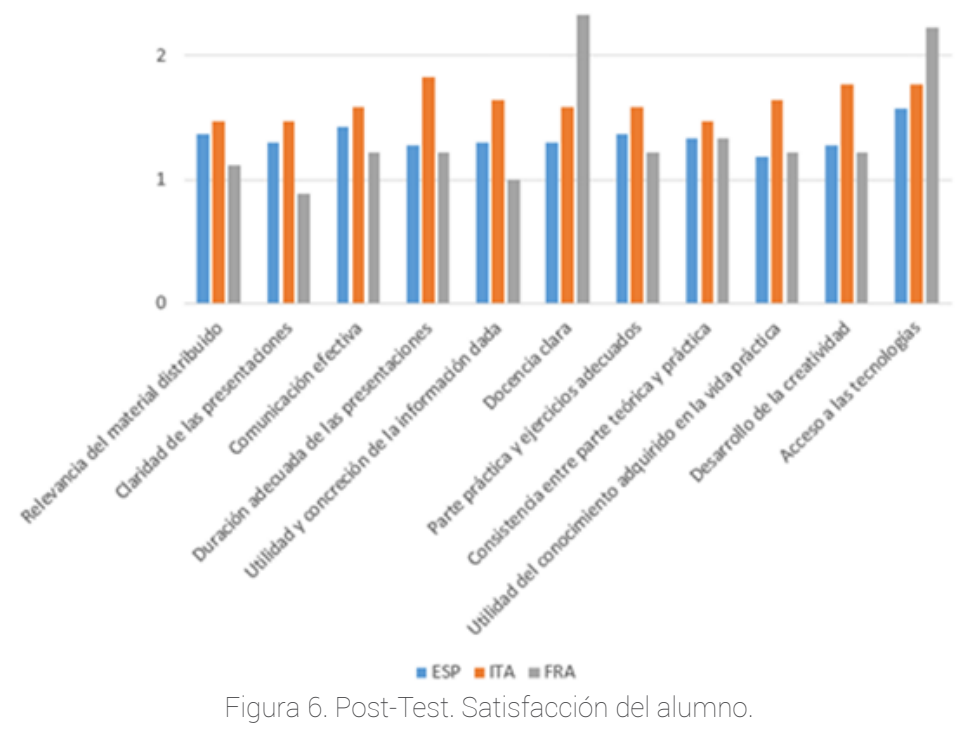

- Los aspectos sugeridos por los alumnos con una mayor necesidad de mejora en cursos futuros han sido de nuevo bastante heterogéneos:

- Mientras en España, el 54\% de los alumnos opinan que es necesario mejorar la comunicación efectiva y el sistema docente, en Italia los dos aspectos más indicados han sido, con un 64\%, la claridad de las presentaciones y con un 58\% la consistencia entre partes teóricas y prácticas, mientras que por encima del índice del 50\% de citación en Francia se sitúa con un 55\% de nuevo la claridad de las presentaciones y el desarrollo de la creatividad. 


\section{Discusión y Conclusiones}

Tal y como previamente se había comentado, la realización de este tipo de proyectos conlleva inicialmente toda una serie de retos: una organización compleja entre socios del proyecto con diferentes perfiles, un encaje de la dedicación del mismo dentro de la planificación de cada organización y un trabajo orientado a que el alumno, eje central de la propuesta, obtenga una mejor formación para conseguir una más rápida y mejor incorporación al mundo laboral. Este proyecto no está siendo ajeno a estas dificultades, destacando cambios organizativos en la dirección de una escuela, la renuncia de participación de otra escuela una vez adjudicado el proyecto y el retraso en la autorización por parte de la ente gestora de la Comisión Europea con los trámites de ambos cambios. Estos problemas burocráticos han conllevado la compactación de un proyecto de tres años, en tan solo dos, generando un flujo de trabajo mucho más intenso. Fruto de la nueva planificación con una temporización más compacta, las escuelas han tenido que realizar un esfuerzo y buscar nuevos recursos para poder gestionar e implementar los cursos. Un ejemplo al respecto ha sido la necesidad de añadir nuevas horas docentes y de tutoría en sus planes académicos, aspectos que ya en las primeras entrevistas realizadas con alumnos y profesores para la evaluación cualitativa final, se posicionan como condicionantes negativos de los resultados obtenidos, especialmente en el primer caso de estudio, que es el que presentamos en el artículo. Con el fin de mejorar los resultados del segundo curso, actualmente en realización, se han articulado nuevos sistemas para que la propuesta no impacte en un aumento de las horas escolares y de dedicación del profesorado, lo cual genera, como hipótesis a comprobar en un futuro, una mejora sustancial en el grado de satisfacción de los alumnos.

Analizando los datos parciales (ya que en este trabajo solo se han tenido en cuenta el test de perfil tecnológico) obtenidos del Pre-Test, encontramos dos aspectos demográficos que pueden influencian los resultados: la muestra de estudiantes por país y su distribución por género. Igualar el número y género de los alumnos debe conllevar una reducción de la desviación estadística de las variables estudiadas, siendo este aspecto una hipótesis a corroborar en el momento de analizar los datos del segundo curso en realización.

Otro aspecto importante es el perfil de los estudios que cursan los alumnos que han realizado el curso mediante SCC. Mientras el grupo español se está formando como técnicos informáticos, el francés está recibiendo formación en seguridad y riesgos laborales, el grupo italiano se centra en formación administrativa, gestión de finanzas y marketing. Este aspecto es importante, ya que justifica los usos y frecuencias de trabajo con dispositivos informáticos e incluso el conocimiento y habilidades en el uso de tecnologías que se han obtenido en el análisis del Pre-Test. Relacionado con el perfil de los 
estudios y el perfil tecnológico de los alumnos, una de las principales conclusiones del estudio se obtiene de las respuestas ligadas a la formación en TIC. Inicialmente se puede observar en el Pre-Test unos resultados extremadamente bajos en cuanto a la satisfacción que estos alumnos han obtenido en formaciones previas, con un valor promedio de 0.64 sobre 3 . El curso de SCC, y valorando más aspectos ligados a la satisfacción que en el Pre-Test, ha obtenido una valoración global de 1.44 sobre 3, que aunque es baja respecto a lo deseado, supone más de un 100\% de incremento en la valoración de un curso en metodologías TIC. Este valor, cercano al aprobado, supone en parte un logro dadas las problemáticas iniciales y previamente comentadas, así como la dificultad de implementación del curso.

Analizando los datos por países, el grupo español que partía con un perfil tecnológico más elevado es quien ha sido más crítico con la metodología propuesta, mientras que en el extremo contrario, el grupo francés, que tanto por perfil de estudios como por su perfil tecnológico partía de un nivel más bajo, es justamente quien ha valorado más positivamente el curso. En conclusión, la metodología SCC se ha adaptado de forma adecuada a los perfiles de estudiantes que hemos tenido, ayudando a una mejora en la adquisición de competencias y en la satisfacción de los estudiantes a la hora de cursar una materia. No obstante, se antoja crítico mejorar la calidad de los materiales y la forma de impartir la metodología para lograr una respuesta más positiva por parte de dichos alumnos, así como una mejor adaptación del modo de impartir la metodología en el día a día del curso académico de los centros.

\section{Agradecimientos}

El Proyecto Learning4work está financiado por la Unión Europea en el marco del programa Erasmus+ número 2014-1-ES01-KA202-004845.

El presente trabajo solo expresa la opinión y puntos de vista de sus autores y tanto la Agencia Nacional como la Comisión Europea no son responsables de cualquier utilización directa o derivada que se puede realizar a partir de los contenidos presentados en el mismo.

\section{Referencias}

Atkins, D. E., Brown, J. S., \& Hammond, A. L. (2007). A review of the open educational resources (OER) movement: Achievements, challenges, and new opportunities. Retrieved from Menlo Park, CA, USA: http://www.hewlett.org/uploads/files/ReviewoftheOERMovement.pdf 
Bareiss, R. \& Griss, M. (2008, March). A story-centered, learn-by-doing approach to software engineering education. In ACM SIGCSE Bulletin, 40 (1), 221-225. ACM. doi: http://dx.doi. org/10.1145/1352322.1352217

Böhme, K., Doucet, P., Komornicki, T., Zaucha, J. \& Świątek, D. (2011). How to strengthen the territorial dimension of 'Europe 2020'and the EU Cohesion Policy. Report based on the Territorial Agenda, 2020.

Damanpour, F. (1987). The adoption of technological, administrative, and ancillary innovations: Impact of organizational factors. Journal of management, 13(4), 675-688. doi: http://dx.doi. org/10.1177/014920638701300408

De Freitas, S. (2006). Learning in immersive worlds. London: Joint Information Systems Committee.

Edens, K. M. (2000). Preparing problem solvers for the 21 st century through problem-based learning. College Teaching, 48(2), 55-60. doi: http://dx.doi.org/10.1080/87567550009595813

Endedijk, M. D., Vermunt, J. D., Meijer, P. C. \& Brekelmans, M. (2014). Students' development in selfregulated learning in postgraduate professional education: a longitudinal study. Studies in higher education, 39(7), 1116-1138. doi: http://dx.doi.org/10.1080/03075079.2013.777402

Estruch, V., \& Silva, J. (2006). Aprendizaje basado en proyectos en la carrera de Ingeniería Informática. Actas de las XII Jornadas de la Enseñanza Universitaria de la Informática (JENUI 2006), Deusto, Bilbao, 12-14 de julio de 2006 (pp. 339-346).

García-Peñalvo, F. J., García de Figuerola, C., \& Merlo-Vega, J. A. (2010). Open knowledge: Challenges and facts. Online Information Review, 34(4), 520-539. doi: http://dx.doi.org/10.1108/14684521011072963

Hew, K. F. \& Cheung, W. S. (2010). Use of three dimensional (3 D) immersive virtual worlds in K 12 and higher education settings: A review of the research. British journal of educational technology, 41(1), 3355. doi: http://dx.doi.org/10.1111/j.1467-8535.2008.00900.x

Higueras, J. (11 de octubre de 2013). Scenario Centered Curriculum, el learning-by-doing de éxito en La Salle Open University. La Salle Open University. Recuperado de http://blogs.uols.org/mba/ scenario-centered-curriculum-el-learning-by-doing-de-exito-en-uols/

Hintikka, J. (1993). Socratic questioning, logic and rhetoric. Revue internationale de philosophie, 47(184), (1), 5-30.

Hmelo-Silver, C. E. (2004). Problem-based learning: What and how do students learn? Educational psychology review, 16(3), 235-266. doi: http://dx.doi.org/10.1023/B:EDPR.0000034022.16470.f3

King, J. L., Gurbaxani, V., Kraemer, K. L., McFarlan, F. W., Raman, K. S. \& Yap, C. S. (1994). Institutional 
factors in information technology innovation. Information systems research, 5(2), 139-169. doi: http:// dx.doi.org/10.1287/isre.5.2.139

Kolb, D. A. (2014). Experiential learning: Experience as the source of learning and development. FT press. Koopman, P., Choset, H., Gandhi, R., Krogh, B., Marculescu, D., Narasimhan, P., ... \& Steenkiste, P. (2005). Undergraduate embedded system education at Carnegie Mellon. ACM Transactions on Embedded Computing Systems (TECS), 4(3), 500-528. doi: http://dx.doi.org/10.1145/1086519.1086522

Law, N., Pelgrum, W. J. \& Plomp, T. (Eds.). (2008). Pedagogy and ICT use in schools around the world: Findings from the IEA SITES 2006 study (Vol. 23). Springer Science \& Business Media.

Popkewitz, T. S. (1977). The latent values of the discipline-centered curriculum. Theory \& Research in Social Education, 5(1), 41-60. doi: http://dx.doi.org/10.1080/00933104.1977.10505997

Ramírez Montoya, M. S., \& García-Peñalvo, F. J. (2015). Movimiento Educativo Abierto. Virtualis, 6(12), $1-13$.

Romiszowski, A. J. (2004). How's the e-learning baby? Factors leading to success or failure of an educational technology innovation. Educational Technology, 44(1), 5-27.

Savery, J. R. \& Duffy, T. M. (1995). Problem based learning: An instructional model and its constructivist framework. Educational technology, 35(5), 31-38.

Schank, R. C. (1983). Dynamic memory: A theory of reminding and learning in computers and people. Cambridge University Press.

Schank, R. C. (2002). Every curriculum tells a story. International Journal of Cognition and Technology, 1(1), 169-182. doi: http://dx.doi.org/10.1075/ijct.1.1.11sch

Shepperd, J. A. (1993). Productivity loss in performance groups: A motivation analysis. Psychological bulletin, 113(1), 67-81. doi: http://dx.doi.org/10.1037/0033-2909.113.1.67

Terenzini, P. T., Pascarella, E. T. \& Blimling, G. S. (1996). Students' out-of-class experiences and their influence on learning and cognitive development: A literature review. Journal of college student development.

Thistlethwaite, J. E., Davies, D., Ekeocha, S., Kidd, J. M., MacDougall, C., Matthews, P., ... \& Clay, D. (2012). The effectiveness of case-based learning in health professional education. A BEME systematic review: BEME Guide No. 23. Medical teacher, 34(6), e421-e444. doi: http://dx.doi.org/10.3109/014215 9X.2012.680939 